\title{
Myotubularin-related protein 7 activates peroxisome proliferator-activated receptor-gamma
}

\author{
Philip Weidner ${ }^{1}$, Michaela Söhn', Torsten Schroeder', Laura Helm¹, Veronika Hauber', Tobias Gutting1, \\ Johannes Betge ${ }^{1}$, Christoph Röcken², Florian N. Rohrbacher ${ }^{3}$, Vijaya R. Pattabiraman ${ }^{3}$, Jeffrey W. Bode ${ }^{3}$, Rony Seger ${ }^{4}$, \\ Daniel Saar $\mathbb{1}^{5}$, Ariane Nunes-Alves $\mathbb{D}^{5,6}$, Rebecca C. Wade $\mathbb{0}^{5,6,7}$, Matthias P. A. Ebert ${ }^{1}$ and Elke Burgermeister ${ }^{1}$
}

\begin{abstract}
Peroxisome proliferator-activated receptor-gamma (PPARY) is a transcription factor drugable by agonists approved for treatment of type 2 diabetes, but also inhibits carcinogenesis and cell proliferation in vivo. Activating mutations in the Kirsten rat sarcoma viral oncogene homologue (KRAS) gene mitigate these beneficial effects by promoting a negative feedback-loop comprising extracellular signal-regulated kinase 1/2 (ERK1/2) and mitogen-activated kinase kinase 1/2 (MEK1/2)-dependent inactivation of PPARY. To overcome this inhibitory mechanism, we searched for novel posttranslational regulators of PPARY. Phosphoinositide phosphatase Myotubularin-Related-Protein-7 (MTMR7) was identified as cytosolic interaction partner of PPARY. Synthetic peptides were designed resembling the regulatory coiled-coil (CC) domain of MTMR7, and their activities studied in human cancer cell lines and C57BL6/J mice. MTMR7 formed a complex with PPARY and increased its transcriptional activity by inhibiting ERK1/2-dependent phosphorylation of PPARY. MTMR7-CC peptides mimicked PPARY-activation in vitro and in vivo due to LXXLL motifs in the CC domain. Molecular dynamics simulations and docking predicted that peptides interact with the steroid receptor coactivator 1 (SRC1)-binding site of PPARY. Thus, MTMR7 is a positive regulator of PPARY, and its mimicry by synthetic peptides overcomes inhibitory mechanisms active in cancer cells possibly contributing to the failure of clinical studies targeting PPARY.
\end{abstract}

\section{Introduction}

The nuclear transcription factor PPAR $\gamma$ has been established as a target in type 2 diabetes for many years. In addition to its lipid lowering and insulin sensitizing properties, pharmacological activation of PPARY shows benefits in malignant and inflammatory human diseases $^{1-3}$, and inhibition of RAS-ERK1/2 signalling was observed in (pre)clinical models ${ }^{4-6}$. It is the latter characteristic that renders the nuclear receptor a promising target in gastrointestinal tumours with frequent, activating mutations in the RAS-ERK1/2 signalling cascade, e.g.

Correspondence: Elke Burgermeister

(elke.burgermeister@medma.uni-heidelberg.de)

'Department of Medicine II, Medical Faculty Mannheim, Heidelberg University, Mannheim, Germany

2Department of Pathology, Christian-Albrechts University, Kiel, Germany

Full list of author information is available at the end of the article in colorectal cancer $(\mathrm{CRC})^{7}$. In this setting, mutations in $R A S$ genes are a major obstacle for effective treatment in advanced disease ${ }^{8}$, and new drugable targets which inhibit RAS-ERK1/2 signalling are needed ${ }^{9}$. However, serious adverse effects limit the long-term monotherapy with PPAR $\gamma$-ligands in metabolic diseases ${ }^{10}$. Nonetheless, combination with chemo- or biological therapies may offer novel strategies against cancer ${ }^{6,11,12}$ Clinical trials investigating the use of PPAR $\gamma$-agonists have yet failed to show sufficient efficacy ${ }^{13,14}$.

One reason for this discrepancy between preclinical and clinical studies may rely on the complex regulation of PPAR $\gamma$ by the RAS-ERK1/2 signalling cascade, which has not been taken into account in any of the before mentioned trials: $\mathrm{we}^{15,16}$ and others ${ }^{17,18}$ demonstrated that downstream effectors of RAS inhibit PPAR $\gamma$, e.g. by ERK1/2-dependent phosphorylation as well as by nuclear

\section{(c) The Author(s) 2020}

(c) Open Access This article is licensed under a Creative Commons Attribution 4.0 International License, which permits use, sharing, adaptation, distribution and reproduction c. in any medium or format, as long as you give appropriate credit to the original author(s) and the source, provide a link to the Creative Commons license, and indicate if changes were made. The images or other third party material in this article are included in the article's Creative Commons license, unless indicated otherwise in a credit line to the material. If material is not included in the article's Creative Commons license and your intended use is not permitted by statutory regulation or exceeds the permitted use, you will need to obtain permission directly from the copyright holder. To view a copy of this license, visit http://creativecommons.org/licenses/by/4.0/. 
export and cytosolic sequestration through MEK1. In addition to this regulatory mechanism, off-target side effects of the first generations of PPAR $\gamma$-agonists even resulted in an increased proliferation rate of tumour and vascular cells, as they involve PPAR $\gamma$-receptor independent ("non-genomic") activation of RAS ${ }^{19}$ and phosphoinositide 3-kinase (PI3K) ${ }^{20}$ signalling, especially at higher dosages.

We therefore hypothesized that the resulting decrease in nuclear transcriptional activity of PPAR $\gamma$, due to its cytosolic sequestration in the presence of an active RAS cascade promotes its targeting to so far unknown cytosolic effectors. Therefore, unravelling novel effectors or modulators of PPAR $\gamma$ could be a promising approach to overcome this obstacle, especially concerning tumours harbouring activating mutations of $R A S$ genes, which are primarily unresponsive to PPARy activation.

In this context, we identified $76 \mathrm{kDa}$ myotubularinrelated protein 7 (MTMR7), a member of the myotubularin (MTM) family of lipid phosphatases, as a novel interaction partner of PPAR $\gamma$. MTMs consist of $\mathrm{N}$ terminal plextrin homology $(\mathrm{PH})$, central protein tyrosine phosphatase (PTP), SET-interaction (SID) and Cterminal coiled-coil (CC) domains ${ }^{21,22}$. Homo- and heterodimerization between a catalytically active member of the family with an enzymatically inactive one, e.g. MTMR6/7/8 with MTMR9, is mediated via the CC domain resulting in an increased enzymatic activity ${ }^{23}$. For murine MTMR7, a truncated $54 \mathrm{kDa}$ isoform has been described lacking this domain ${ }^{24}$. The active enzyme then dephosphorylates phosphatidyl-inositol-3-monophosphate $(\mathrm{PI}(3) \mathrm{P})$ and $-3,5$-bisphosphate $\left(\mathrm{PI}(3,5) \mathrm{P}_{2}\right)$. MTMs are membrane-bound and localize to endosomes, with the exception of MTMR7, being present in a soluble form in the cytoplasm ${ }^{24}$ using free inositol-1,3-bisphosphate (Ins $\left.(1,3) \mathrm{P}_{2}\right)$ as a substrate.

In addition to the previously reported expression of MTMR7 in brain, muscle, liver and kidney ${ }^{24}$, we detected MTMR7 in the gastrointestinal tract ${ }^{25}$. In contrast to other MTMs, characterized as "survival phospha-

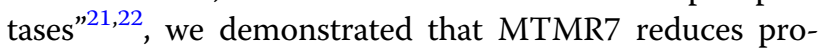
liferation of CRC cells in vitro, even in the presence of activating mutations of KRAS and active insulin signalling, due to inhibition of both RAS-ERK1/2 and PI3K-AKTmTOR signalling ${ }^{25}$.

In the present study, we describe a novel regulatory mechanism of PPAR $\gamma$ which augments its transcriptional activity via its interaction with MTMR7. In addition, we offer new insights into the subcellular distribution of MTMR7 in response to external stimuli and identified the CC domain of MTMR7, by designing and modifying a peptide resembling this domain, as a potential novel pharmacological activator of PPAR $\gamma$ in vitro and in vivo.

\section{Results}

\section{MTMR7 is a cytosolic binding partner of PPARy}

In cancer cells with constitutive activation of RASERK1/2 signalling, PPAR $\gamma$ can be translocated from the nucleus to the cytosol by a previously described MEK1dependent export mechanism ${ }^{15,26}$. However, the function of cytosolic PPAR $\gamma$ is unknown. To identify novel binding partners which may act as regulators or effectors for cytosolic PPAR $\gamma$, a matrix-assisted laser desorption/ionization mass spectrometry (MALDI-MS) protein interaction screen was performed in the aneuploid human CRC cell line SW480, which has mutated alleles of the KRASG12V gene $^{27}$ and a high amount of extra-nuclear PPARy. SW480 cells were disrupted by hypotonic lysis without detergents, a procedure which extracts cytosolic proteins. Coimmunoprecipitation (CoIP) was then performed with PPAR $\gamma$ antibody (Ab) or control IgG followed by detection of precipitated bands by silver staining. Peptides within a band of $54 \mathrm{kDa}$ were coprecipitated by the PPAR $\gamma \mathrm{Ab}$ and identified to correspond to human MTMR7 by MALDI-MS sequencing (Fig. 1a; Tab. S1). Most of the peptides covered the internal and Cterminal part of the enzyme, including the catalytic phosphatase, SID and CC domains.

For detection of the cytosolic complex between endogenous MTMR7 and PPAR $\gamma$, we resorted to HCT116, a human KRASG13D mutated CRC cell line which expresses high amounts of full-length (FL) MTMR7 $(76 \mathrm{kDa})$ protein $^{25}$. Subcellular fractionation (SCF) of normal cycling HCT116 cells was performed, and cytosolic lysates were immunoprecipitated with MTMR7 or PPARY Ab-conjugated or unconjugated beads. Immunoblotting (IB) was performed with MTMR7 Ab (Fig. 1b). MTMR7 Abs were directed against the C-terminal region of the enzyme and pulled down a $76 \mathrm{kDa}$ band corresponding to the FL protein.

For detection of ectopic proteins, we employed HEK293T cells which have low amounts of endogenous FL MTMR7 (76 kDa) protein ${ }^{25}$. Cells were transiently cotransfected with expression plasmids encoding for GFPPPARY and MTMR7 (FL) protein for $24 \mathrm{~h}$. After SCF, CoIP from cytosolic lysates was performed using MTMR7 or PPAR $\gamma$-Ab-conjugated beads. IB against MTMR7 detected an $80 \mathrm{kDa}$ band, representing GFP-PPAR $\gamma$ after MTMR7 pulldown (Fig. 1c upper panel). For CoIP performed with PPAR $\gamma$ Ab-conjugated beads, a $76 \mathrm{kDa}$ overexpressed band was detected by the MTMR7 Ab (Fig. 1c lower panel). To corroborate the findings on this interaction, proximity ligation assay (PLA) was conducted in HEK293T cells after a $24 \mathrm{~h}$ transfection with GFPMTMR7 (FL) (Fig. 1d) or GFP-PPARY (Fig. 1e) expression plasmids, respectively. Thereafter, cells were subjected to immunofluorescence imaging, and colocalization of MTMR7 and PPAR $\gamma$ proteins was visualized as pink dots. 


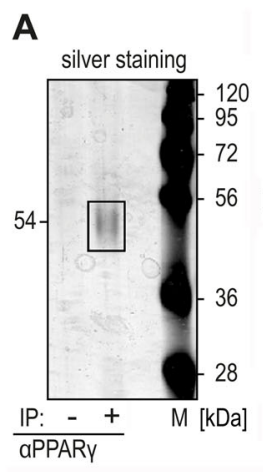

D

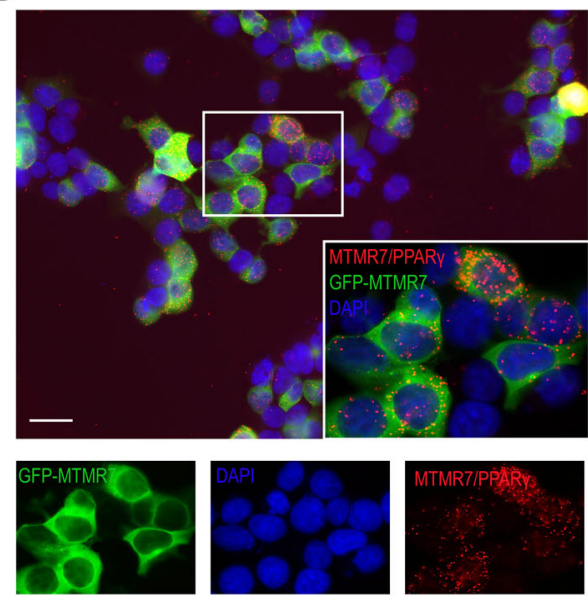

B

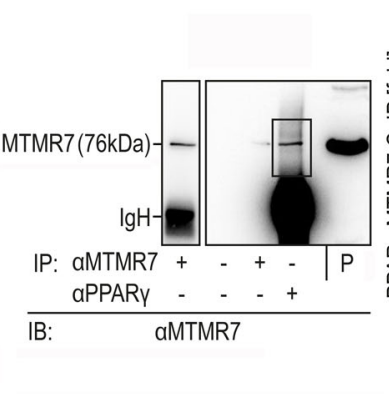

C
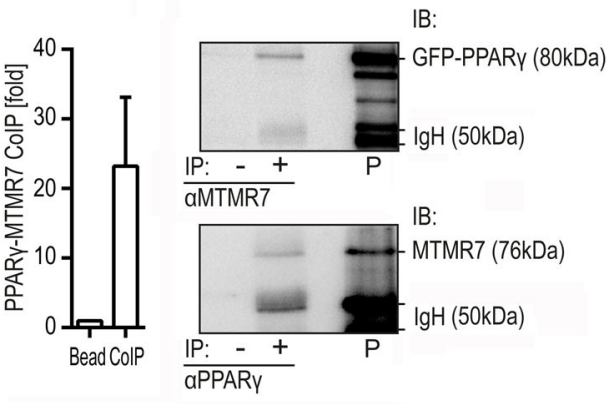

E

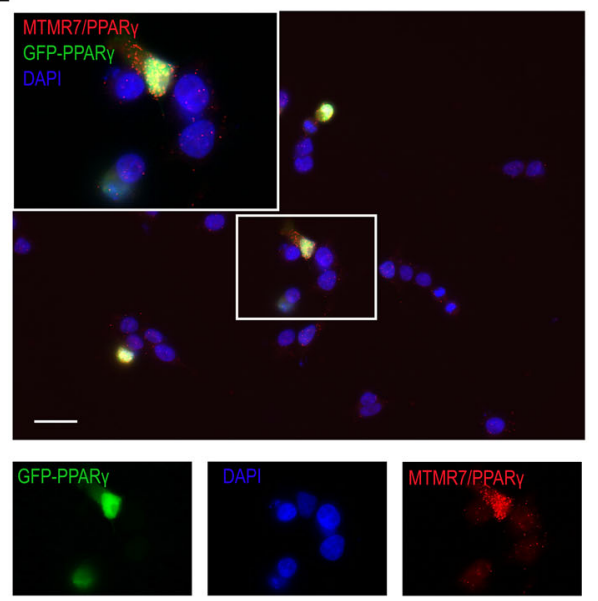

Fig. 1 Identification of MTMR7 as a cytosolic interaction partner of PPARy. a Coimmunoprecipitation (ColP) was performed on cytosolic lysates of SW480 cells using PPARY Ab (\#7196) or lgG (bead control) followed by detection of precipitated bands by silver staining. MALDI-MS sequencing of a $54 \mathrm{kDa}$ band precipitated by PPARY Ab contained peptides from human MTMR7 (Tab. S1). M = marker. b ColP of endogenous proteins in cytosolic lysates of HCT116 cells using PPARY (lane 4: \#7196) or MTMR7 (lane 1: \#121222; lane 3: \#51145) Abs for IP and IB, respectively. Representative gels and quantitative analyses are shown. Absolute O.D. values from bands in gels are shown as -fold \pm S.E. compared with bead control ( $n=3$ replicates). $\mathrm{P}=$ input. c CoIP of ectopic proteins. HEK293T cells were transiently co-transfected with GFP-PPARY and MTMR7 (FL) expression plasmids for $24 \mathrm{~h}$. ColP was performed on cytosolic lysates using PPARY (\#1196) or MTMR7 (\#121222) Abs, respectively. Representative gels are shown. P = input. d, e Proximity ligation assay (PLA). HEK293T cells were co-transfected with untagged PPARY and GFP-MTMR7 FL (d) or untagged MTMR7 FL and GFPPPARY (e) expression plasmids for $24 \mathrm{~h}$, respectively. Immunofluorescence microscopy was performed using PPARY (\#2435) (d), MTMR7 (\#121222) (e) and GFP Abs. Representative images are shown. Scale bar $=20 \mu \mathrm{m}$. Colour legend: red $=$ PPARY/MTMR7 complex; green $=$ GFP; blue $=$ nuclei $(\mathrm{DAPI})$; original magnification $\times 630$.

These data indicated that MTMR7 forms a complex with PPARY.

\section{Nuclear translocation of MTMR7 in response to growth factors and PPARY-agonist}

To interrogate the subcellular distribution of MTMR7 and PPAR $\gamma$, immunofluorescence microscopy was performed on normal cycling HCT116 cells expressing high levels of endogenous MTMR7 protein (Fig. 2a) ${ }^{25}$. In this setting, predominant cytosolic localization could be detected ( $p=0.057$ nuclear "N" vs. cytosolic "C", Mann-Whitney test, $n=4$ replicates). In addition, we evaluated formalin-fixed paraffin-embedded (FFPE) tissue specimens from CRC patients ( $n=67$ cases) with regard to MTMR7 expression and subcellular localization by means of immunohistochemistry (IHC). Of these patients, 41 (61\%) showed a positive MTMR7 staining. In 19 (46\%) of these MTMR7 positive cases, a nuclear staining was detected (Fig. 2b).

For 25 samples, an additional staining for PPAR $\gamma$ was observed, 16 (64\%) of which exhibited a positive nuclear staining for PPARY (Fig. 2c). Nuclear PPAR $\gamma$ staining was associated with nuclear localization of MTMR7 ( $p=0.04$, Fisher's exact test, $n=67$ patients).

To further study stimulation-dependent nuclear translocation of MTMR7, immunofluorescence microscopy was performed in HCT116 cells expressing high endogenous MTMR7 FL protein. Cells were serum-deprived for $16 \mathrm{~h}$, followed by incubation with PPAR $\gamma$-agonist rosiglitazone (rosi) $(1 \mu \mathrm{M}, \mathrm{R} 1)$, epidermal growth factor 


\section{A}

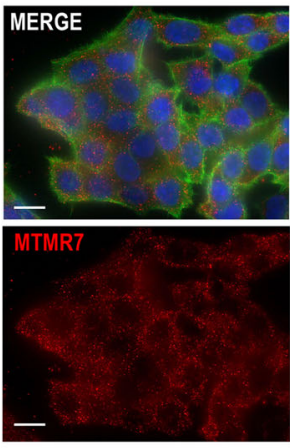

B

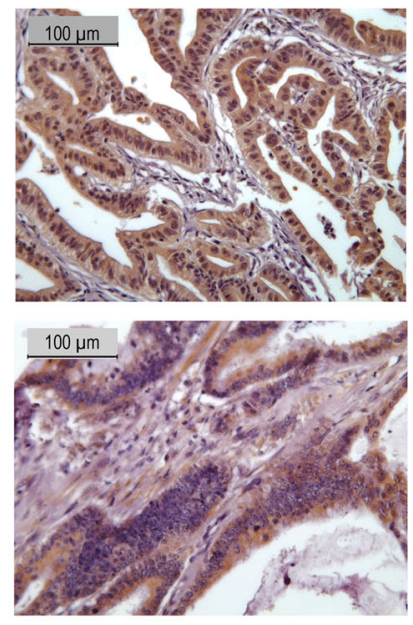

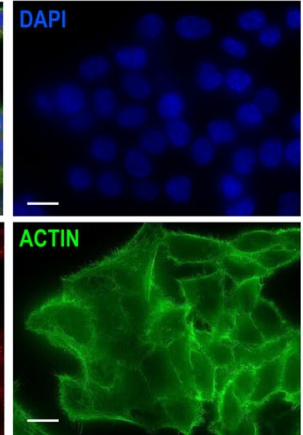

C

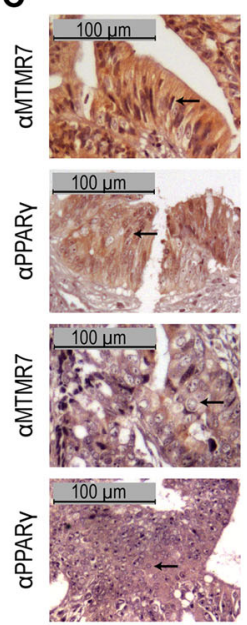

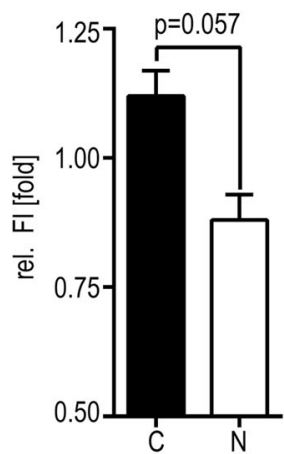

nuc. MTMR7 neg.

$\square$ nuc. MTMR7 pos.

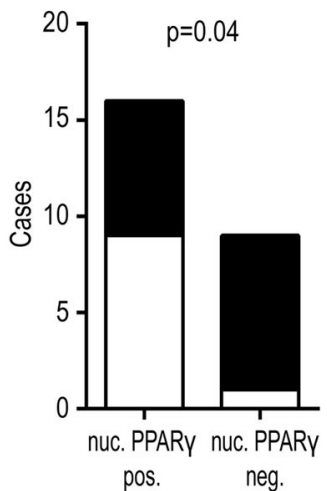

Fig. 2 Subcellular distribution of MTMR7 protein variants. a Localisation of MTMR7. Immunofluorescence microscopy was performed using MTMR7 Ab (\#121222) in HCT116 cells, expressing endogenous MTMR7 protein. Left: Representative images. Right: Quantification of nuclear ("N") and cytoplasmatic ("C") fluorescence intensity (FI) revealed a predominant cytoplasmatic localization of MTMR7. Data are -fold FI \pm S.E. compared with control ( $p=0.057 \mathrm{~N}$ vs. C, Mann-Whitney test, $n=4$ replicates). Scale bar $=10 \mu \mathrm{m}$. Colour legend: red $=$ MTMR7, green $=$ actin (phalloidin), blue $=$ nuclei (DAPI); original magnification $\times 630$. b Localisation of MTMR7 in human CRC patients' tissues ( $n=67$ cases). Immunohistochemistry (IHC) using MTMR7 Ab (\#121222) revealed a subset of patients with positive nuclear MTMR7 staining (upper panel), whereas it was detectable mainly in the cytosol of other cases (lower panel). Representative images are shown; original magnification $\times 200$. c Reduced nuclear localization of PPARY is associated with increased cytosolic localization of MTMR7. IHC staining with MTMR7 (\#121222) and PPARY (\#7196) Abs was performed on matched human CRC patients' tissue specimens. Left: Representative images of cases with (upper two panels) or without (lower two panels) nuclear MTMR7 and PPARy staining are shown; representative nuclei are marked by arrows. Right: Quantitative analyses evinced that lower numbers of PPARY ${ }^{+}$nuclei correlated with reduced MTMR7 ${ }^{+}$nuclei $(p=0.04$, Fisher's exact test, $n=67$ cases). Reduction of nuclear PPARy staining was defined as $<30 \%$ positive nuclei.

(EGF) $(50 \mathrm{ng} / \mathrm{ml})$ or foetal calf serum (FCS) $(20 \% \mathrm{v} / \mathrm{v})$ for $1 \mathrm{~h}$ (Fig. 3a, b). Upon stimulation with either FCS or rosi, an increase in the nuclear fluorescence intensity (FI) was detected (FCS $p=0.0173$, R1 $p=0.0431$ ), while there was a trend for a predominant cytoplasmic localization in starved cells $(p=0.11)$ (N vs. C, two-way ANOVA, Sidak's multiple comparisons test, $n=3$ replicates). Thus, endogenous MTMR7 translocates from the cytosol to the nucleus in response to serum or PPAR $\gamma$-activation.

However, this method did not discriminate between FL and small isoforms of MTMR7 $7^{24,25}$. We therefore studied subcellular localization in HEK293T cells which had low endogenous MTMR7 FL protein. Cells were starved for
$16 \mathrm{~h}$ and then stimulated with serum or PPAR $\gamma$-agonist, followed by SCF and IB against MTMR7 (Fig. 3c). Upon incubation with $20 \%(\mathrm{v} / \mathrm{v})$ FCS or rosi $(1 \mu \mathrm{M}, \mathrm{R} 1)$, the endogenous, truncated MTMR7 isoform $(54 \mathrm{kDa})$ accumulated in the nuclear fraction ( $\mathrm{N}$ vs. C: FCS $p=0.0440$, R1 $p=0.0008$; two-way ANOVA, Sidak's multiple comparisons test, $n=3$ replicates). All Abs used in the present study recognized the C-terminal region of the MTMR7 FL protein (Tab. S2), indicative of a stimulus-dependent mobility of both isoforms.

To characterize the subcellular localization of MTMR7 in situ, we cultivated patient-derived organoids (PDOs) of four patients harbouring activating KRAS mutations and 

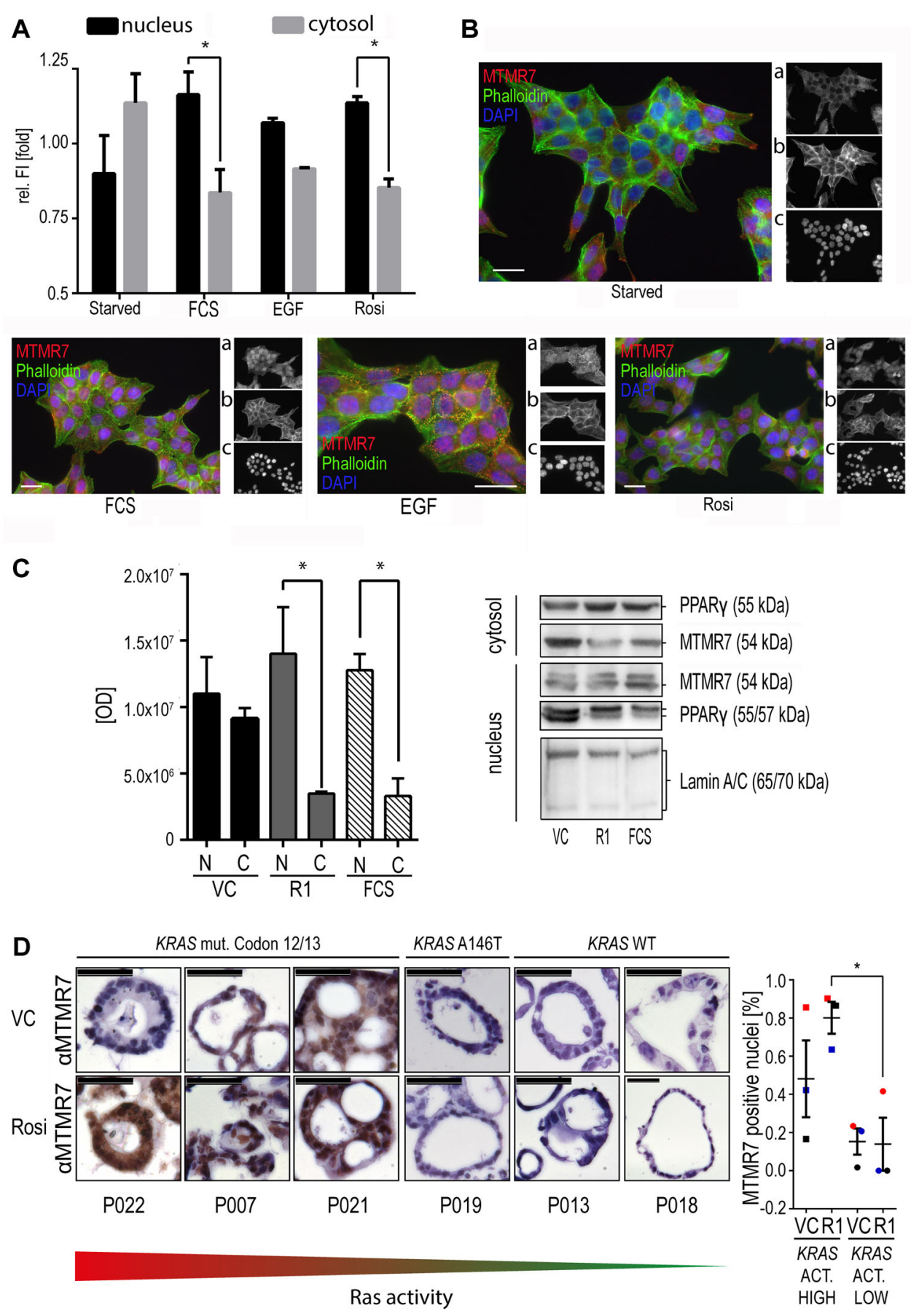

Fig. 3 (See legend on next page.)

two patients with wild-type (WT) KRAS. The PDOs were incubated for $48 \mathrm{~h}$ in medium containing $1 \mu \mathrm{M}$ rosi or vehicle control (VC, DMSO) (Fig. 3d): On baseline, 4 of the 6 PDOs showed a positive MTMR7 staining, 3 of which had an activating KRAS codon 12 or 13 mutation.
Remarkably, the rosi-stimulated PDOs showed an increase in nuclear MTMR7. This effect was more pronounced in the PDO lines with the activating KRAS mutations of codon 12 or 13 as compared with KRAS WT PDO lines or the line with the A146T mutation $(p=0.016$ 


\begin{abstract}
(see figure on previous page)
Fig. 3 Nuclear translocation of MTMR7 in response to growth factors and PPARy-agonist. a Immunfluorescence microscopy. HCT116 cells were serum-deprived for $16 \mathrm{~h}$ ("starved") and subsequently stimulated with serum ( $20 \% \mathrm{v} / \mathrm{v} \mathrm{FCS})$, EGF ( $50 \mathrm{ng} / \mathrm{ml})$ or rosi $(1 \mu \mathrm{M})$ for $1 \mathrm{~h}$, followed by fixation and staining of endogenous MTMR7 (Ab: \#121222). FI signals from the nuclear and cytosolic compartments were normalized to the overall Fl. Data are -fold FI \pm S.E. ( ${ }^{*} p<0.05 \mathrm{~N}$ vs. C, two-way ANOVA, Sidak's multiple comparisons test, $n=3$ replicates). While there was no difference in the distribution of endogenous MTMR7 in starved cells, $1 \mathrm{~h}$ stimulation with serum, EGF or rosi increased nuclear MTMR7 Fl. b Representative images from (a) using Abs against MTMR7 (red, a), actin (phalloidin) (green, b), nuclei (DAPI) (blue, c). Scale bars $=20 \mu$ m. Original magnification: $\times 630$. c Endogenous MTMR7 translocates into the nucleus in response to growth factors and PPARY-agonist. HEK293T cells were serum-deprived for $16 \mathrm{~h}$ followed by incubation with vehicle control (VC; DMSO), rosi (R; $1 \mu \mathrm{M})$ or 20\% (v/v) FCS for $1 \mathrm{~h}$. Thereafter, cells were subjected to SCF and IB for MTMR7 (Ab: \#150458). Representative gels and quantitative analyses. Mean O.D. values \pm S.E. from bands in gels are shown $\left(^{*} p<0.05 \mathrm{~N}\right.$ vs. C, two-way ANOVA, Sidak's multiple comparisons test, $n=3$ replicates). $C=$ cytoplasm; $N=$ nucleus. $\mathbf{d}$ MTMR7 translocates into the nucleus in patient-derived organoids (PDOs). Cell lines from six different CRC patients were cultivated for $48 \mathrm{~h}$ in medium containing VC or $1 \mu \mathrm{M}$ rosi (R1). Left panel: IHC against MTMR7 (Ab: \#9406043) was performed, and 4 of 6 PDO lines showed a positive MTMR7 staining at baseline which increased upon rosi stimulation. In 2 of 3 PDO lines with activating KRAS mutations, MTMR7 staining intensity increased after rosi stimulation. Each PDO is labelled by its patient ID. The KRAS-mutation type is given above the representative images. Right panel: The percentage of $\mathrm{MTMR7}^{+}$nuclei after rosi stimulation was higher in PDO lines with activating codon 12 and 13 mutations of KRAS ( $p=0.016$ high vs. Iow KRAS activity, two-way ANOVA, Holm-Sidak's multiple comparisons test, $n=6$ cases). Pre- and post-stimulation pairs are coded in the same colour.
\end{abstract}

high vs. low KRAS activity, two-way ANOVA, Holm-Sidak's multiple comparisons test, $n=6$ cases). Thus, PPAR $\gamma$-agonist facilitates nuclear accumulation of MTMR7 in vitro and ex vivo in patient-derived cancer stem cells.

\section{MTMR7 increases nuclear transcriptional activity of PPAR $\gamma$}

To assess, whether MTMR7 alters the transcriptional activity of PPAR $\gamma$, we performed reporter gene assays using a luciferase plasmid with a PPAR $y$-responsive enhancer element (PPRE) in front of a basal promoter. HCT116, SW480 and HEK293T cells were transfected with empty vector (EV) or MTMR7 (FL) expression plasmid followed by stimulation with rosi $(0.1-10 \mu \mathrm{M})$ for $48 \mathrm{~h}$ (Fig. 4a-c). MTMR7 overexpression increased basal (HCT116 \& SW480: $p<0.0001$ ) and ligand-dependent (HCT116 \& SW480: $p<0.0001$; HEK293T: $1 \mu \mathrm{M}: p=$ $0.0295,10 \mu \mathrm{M}: p=0.0425)$ reporter gene activity compared with EV control (replicates: HCT116: $n=12$; SW480: $n=6$; HEK293T: $n=12$; two-way ANOVA, Sidak's multiple comparisons test, EV vs. MTMR7).

To explore whether these results correlate with increased PPAR $\gamma$-target gene expression, in silico analysis using the cBioportal database of cancer genomics was conducted $^{28}$. MTMR7 mRNA expression $\leq 1$ S.D. of the mean positively correlated with a reduced expression of a broad set of PPAR $\gamma$-target genes in the CRC dataset provided by TCGA Nature 2012 (Fig. 4d). This finding was reproduced in the dataset provided by the Pancancer Atlas (not shown).

To elucidate a possible mechanism underlying the observed increased PPAR $\gamma$-activity in presence of MTMR7, we performed SCF followed by IB for phosphorylated PPAR $\gamma$, using an Ab specific for the bona fide ERK1/2-phosphorylation site at serine 82/84. To this end, HEK293T cells were transfected with EV or MTMR7 (FL) and treated with vehicle control (VC), 20\% (v/v) FCS or
$1 \mu \mathrm{M}$ rosi (R1) for $24 \mathrm{~h}$, respectively (Fig. 4e). After MTMR7 overexpression, a trend for reduced amount of nuclear, phosphorylated (i.e. inactivated) PPAR $\gamma$ was detectable. Hence, MTMR7 seems to increase PPAR $\gamma$ activity indirectly by reducing its inhibitory phosphorylation through the RAS-MEK1/2-ERK1/2 pathway $^{16}$.

\section{MTMR7 inhibits PPAR $\gamma$-agonist-mediated ERK1/2 activation}

Since at least some of the off-target side effects of rosi (and related glitazones) may be mediated by the aberrant activation of MEK1/2-ERK1/2 signalling ${ }^{29,30}$, we investigated the effect of MTMR7 overexpression on rosi-mediated ERK1/2 phosphorylation. To this end, HCT116 cells were transiently transfected with EV or MTMR7 (FL), serum-deprived for $16 \mathrm{~h}$ and subsequently incubated with $10 \mu \mathrm{M}$ rosi, a concentration higher than its $\mathrm{IC}_{50}$ and shown to stimulate ERK $1 / 2^{30}$. This effect was abrogated by overexpression of MTMR7, leading to reduced amounts of phosphorylated ERK1/2 ( $p=0.0449$, Kruskal-Wallis test), particularly after 10 min of rosi stimulation ( $p=0.0392 \mathrm{EV}$ vs. MTMR7, Dunn's multiple comparisons test) $(n=3$ replicates $)$ (Fig. 4f). A similar result was shown for SW480 cells (Supplementary Fig. 1A).

\section{Design of MTMR7-CC mimicry peptides}

The main regulatory mechanism of catalytically active MTMs is the formation of homo- or heterodimers with partner MTMs and effector proteins by means of the C-terminal coiled-coil (CC) domain ${ }^{31}$. We therefore hypothesized that the CC domain of MTMR7 is the region of the protein responsible for the interaction with PPAR $\gamma$. To test this idea, we designed a synthetic peptide to substitute for the MTMR7 FL protein. There is no crystal structure of the MTMR7's CC domain or any other MTM available. However, Kim et al. identified and 


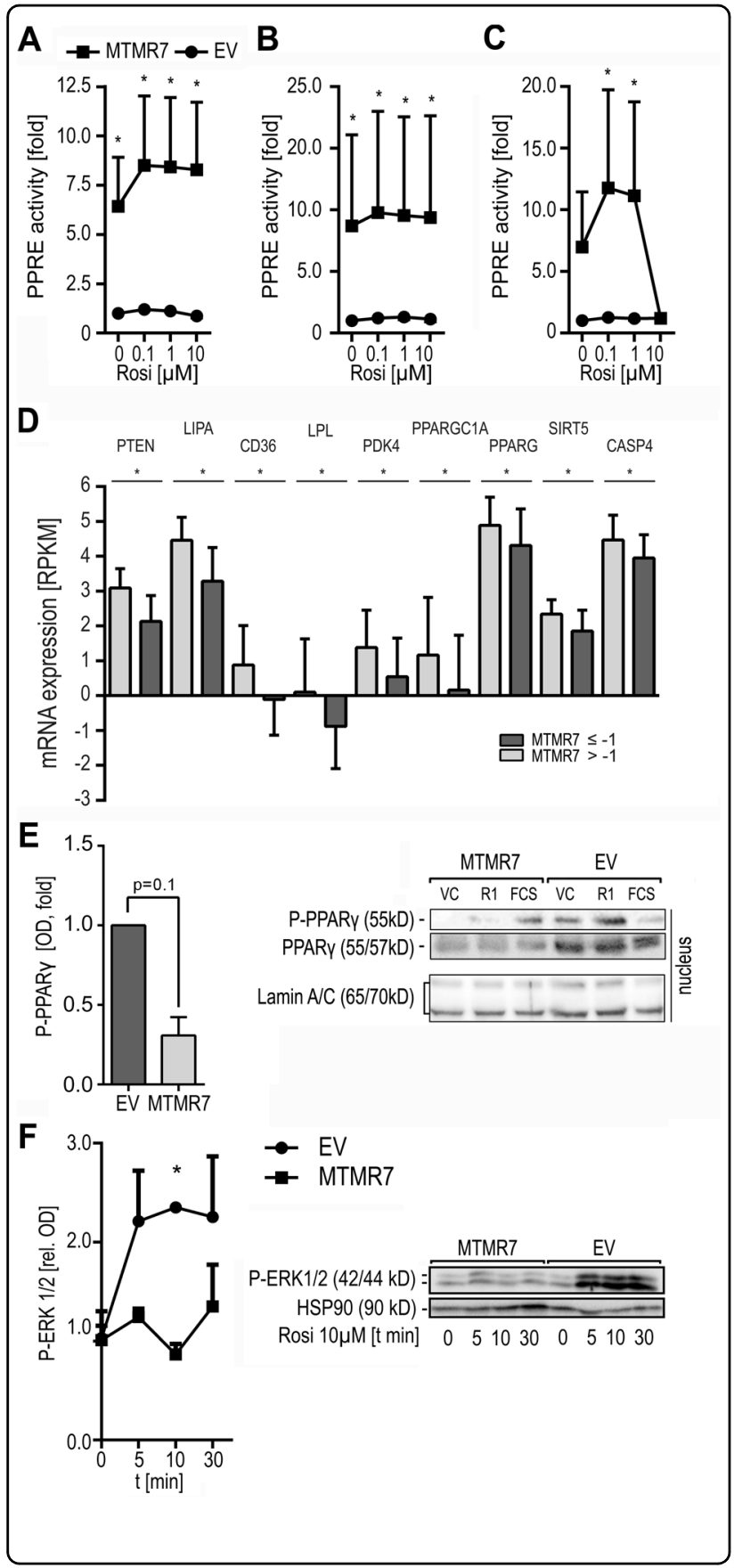

characterized the CC domain of MTMR2 ${ }^{32}$. Performing multiple alignment analysis of the amino acid (aa) sequences of MTMR7, MTMR2, MTMR6, MTMR8 and MTMR9, we identified a stretch of 30 amino acids at the C-terminus of MTMR7 (Fig. 5a1).

This leucine-rich region showed a high coil-forming capacity using NCOILS version 1.0 (ExPASy; Supplementary Fig. 1B). Therefore, we used this region as a basis for the design of a mimicry peptide (PEP) of the MTMR7CC. Pepwheel, provided by EMBOSS, predicted the
Fig. 4 MTMR7 increases the transcriptional activity of PPARy. a-c MTMR7 promotes PPARY-dependent reporter gene expression. HCT116 (a), SW480 (b) and HEK293T (c) cells were transfected with empty vector (EV) or MTMR7 FL expression plasmid, respectively, together with PPARy-reporter plasmid (PPRE-luc) for $24 \mathrm{~h}$ before stimulation with rosi $(0-1 \mu \mathrm{M})$ for additional $24 \mathrm{~h}$. Luciferase activity was measured in total cell lysates, normalised to protein content and calculated as -fold \pm S.E. compared with control $\left(^{*} p<0.05\right.$ EV vs. MTMR7, two-way ANOVA Sidak's multiple comparisons test; replicates: HCT116 $n=12$; SW480 $n=6$; HEK293T $n=12$ ). d Downregulation of MTMR7 mRNA is associated with decreased expression of PPARY target genes. In silico analysis using the Colorectal Adenocarcinoma (TCGA, Nature 2012) dataset provided by the cBioPortal database for Cancer Genomics revealed a downregulation $\geq 1$ S.D. in 38 of 182 CRC patients analysed. This downregulation was associated with reduced "Reads per kilo base per million mapped reads" (RPKM) counts of PPARy target genes. Data are means \pm S.D. and significances $\left({ }^{*} p<0.05\right.$ high vs. Iow MTMR7 mRNA) as disclosed by the provider. e MTMR7 reduces inhibitory phosphorylation on serine 82/84 on PPARY1. HEK293T cells were transfected with MTMR7 (FL) for $6 \mathrm{~h}$, followed by serum-deprival for $16 \mathrm{~h}$ and subsequent stimulation with vehicle (VC, DMSO), rosi (1 $\mu \mathrm{M}, \mathrm{R} 1)$ or $20 \%$ (v/v) FCS for 60 min, followed by SCF. IB of unphosphorylated and phosphorylated PPARY was performed. Data are absolute O.D. values calculated as -fold \pm S.E. compared with EV control ( $p=0.1$ EV vs. MTRM7, Mann-Whitney test, $n=3$ replicates). f, MTMR7 abrogates rosi-mediated activation of ERK1/2. HCT116 cells were transfected with MTMR7 FL plasmid for $6 \mathrm{~h}$, followed by serumdeprival for $16 \mathrm{~h}$ and stimulation with rosi $(10 \mu \mathrm{M})$ for the times indicated (min). IB on total cell lysates detected reduced ERK1/2 phosphorylation in presence of MTMR7. Data are absolute O.D. values normalized to HSP90 calculated as -fold \pm S.E. $(p=0.0449$, Kruskal-Wallis test, subgroup analysis: $10 \mathrm{~min}, p=0.0392 \mathrm{EV}$ vs. MTMR7, Dunn's multiple comparisons test; $n=3$ replicates).

selected sequence to form an amphipathic $\alpha$-helix (Supplementary Fig. 1C). In addition, a modified peptide (MP), consisting of the same amino acid residues in a scrambled order, but containing a canonical LXXLL coactivator motif for nuclear receptors ${ }^{33}$ (including PPARY) was designed (Fig. 5a2). Both peptides were myristoylated at the $\mathrm{N}$-terminus and amidated at the $\mathrm{C}$-terminus to enhance uptake into cells and minimize proteolysis.

\section{MTMR7 mimicry peptides activate PPARy in vivo and in vitro}

To determine the effect of the peptides on the transcriptional activity of PPAR $\gamma$, HEK293T cells were transfected with the PPRE-luciferase reporter plasmid for $24 \mathrm{~h}$. Thereafter, treatment with vehicle control (VC), MP or PEP (both at $1 \mu \mathrm{M}$ ) was performed in presence or absence of rosi $(1$ and $10 \mu \mathrm{M})$. As for overexpression of MTMR7 FL protein, treatment with both peptides increased the transcriptional activity of PPARY (Fig. 5b, $" p<0.05$ vs. VC, two-way ANOVA, Holm-Sidak's multiple comparisons test, $n=7$ replicates). A similar effect was observed in HCT116 $(n=3)$ and SW480 $(n=5)$ replicates (" $p<0.05$ vs. VC, one-sample $t$ test). Treatment 
A 2 MTMR7-CC Peptide

LMAVKEETQQLEELEALEERLEKIQKVQI $\mathrm{WWi}_{\text {- }}$

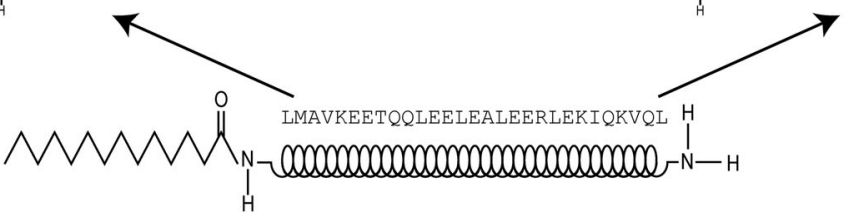

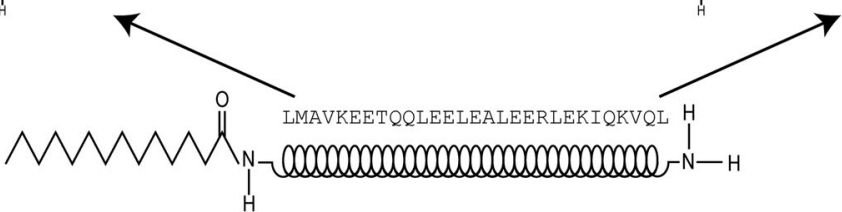

B

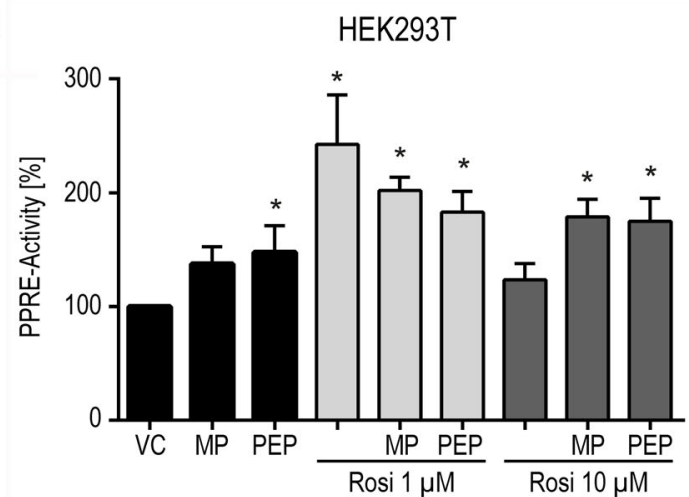

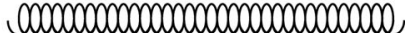

abc def gabc def gabc def $g$ abc def $g$

ICI|hMTMR7 509 KGMMPRQSVTDYIMAVKEETQQDEEEAEATEERIEKIQKVQL . . . . QKVQINCTKVKS

lcl|hMTMR2 585 PRMKRQEPIHNRYKELLAKRAEIQKKVEELQREISNR $\ldots \ldots \ldots \ldots \ldots \ldots \ldots$

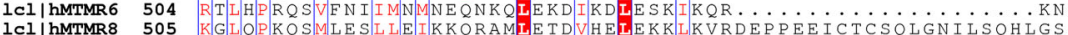

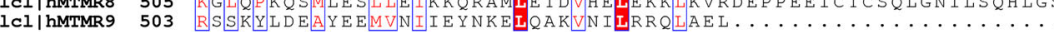
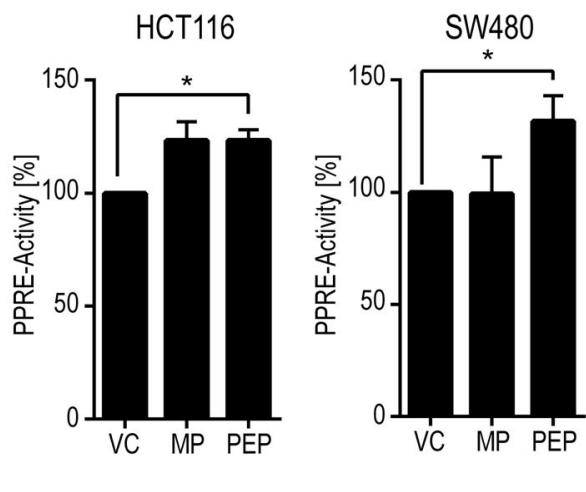

C
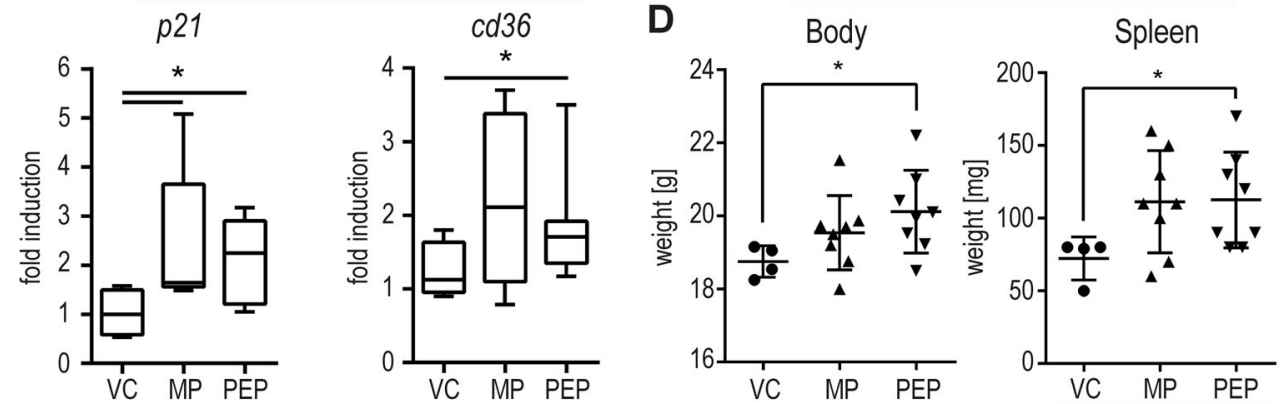

Fig. 5 Design of MTMR7-CC mimicry peptides which activate PPARy in vitro and in vivo. a, 1: Alignment of the amino acid (aa) sequences of the coiled-coil (CC) domains of MTMR7, 2, 6, 8 and 9. The heptad repeat is denoted with the letters a- $g$, with the leucines of MTMR2 residing in the $d$ position. Residues of MTMR2 required for heterodimerization are marked green ${ }^{32}$. This figure was drawn using ESPript (http://espript.ibcp.fr).

a, 2: MTMR7 harbours a 30 aa sequence of MTMR7 with high coil-forming capacity. The N-terminus was modified by myristoylation, the C-terminus was amidated. A modified peptide consisting of the same aa sequence in a scrambled order, but with a PPARY coactivator LXXLL motif, was designed as control. b MTMR7-CC peptides promote transcriptional activity of PPARY. HEK293T, HCT116 and SW480 cells were transfected with PPARYluciferase reporter plasmid (PPRE-luc) for $24 \mathrm{~h}$ before incubation with $1 \mu \mathrm{M}$ of a peptide mimicking the coiled-coil (CC) domain of MTMR7 (PEP) and a modified peptide (MP) with a scrambled a-helix composition but a preserved LXXLL coactivator motif in presence or absence of rosi (1 \& $10 \mu M)$ for additional $24 \mathrm{~h}$. Luciferase activity normalised to protein content was calculated as $\% \pm$ S.E. compared with vehicle control $\left({ }^{*} p<0.05 \mathrm{Vs}\right.$. VC, HEK293T: two-way ANOVA, Holm-Sidak's multiple comparisons test, $n=7$ replicates; HCT116: one-sample $t$ test, $n=3$ replicates, SW480: one-sample $t$ test; $n=5$ replicates). c PEP and MP activate PPARy-target genes in vivo. C57BL6/J mice (pCEA-SV40-Tag) were treated for 14 days with MP, PEP (both at $30 \mathrm{mg} / \mathrm{kg}^{*} \mathrm{~d}$, four times a week) or vehicle control (VC, DMSO) by intraperitoneal injection, respectively. RT-qPCRs detecting PPARY-target genes were performed on RNA extracted from snap-frozen whole tissue samples derived from the distal colon. MP and PEP treatment increased P21(Cip 1 Wafi) and $C d 36$ mRNA expression. CT-values were normalized to B2m and calculated as -fold \pm S.E. and compared with controls $\left({ }^{*} p<0.05\right.$ vs. VC, Mann-Whitney test, VC: $n=4$, MP: $n=8$, PEP: $n=8$ mice per group). $\mathbf{d}$ PEP and MP increase spleen weight and induce body weight gain, thereby mimicking a rosilike phenotype ${ }^{60}$ : pCEA-SV40-Tag mice were treated as above. Body and spleen weights were measured after 14 days of therapy. Data are means \pm S.E. compared with controls ( ${ }^{*} p<0.05$ Vs. VC, Mann-Whitney test, VC: $n=4$, MP: $n=8$, PEP: $n=8$ mice per group). 
A

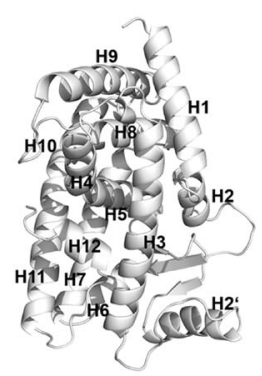

D

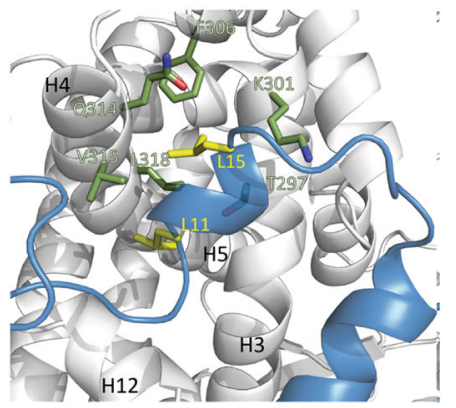

B

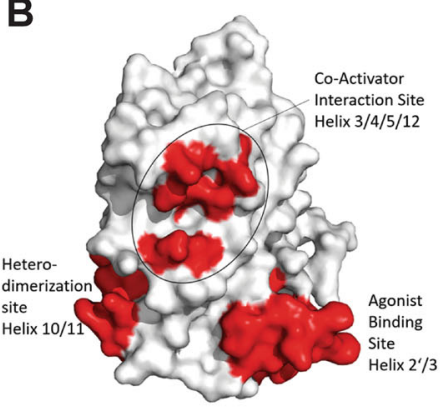

E

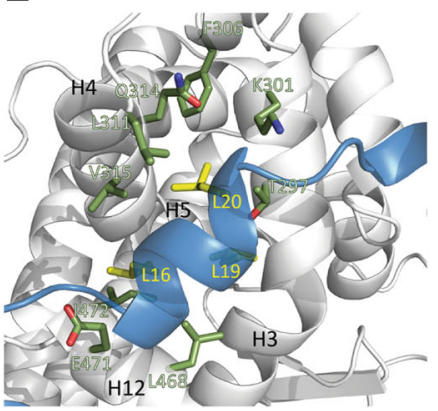

C

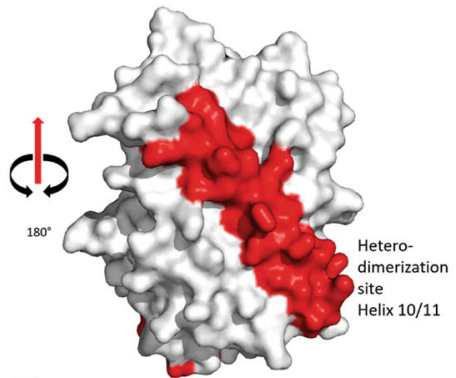

$\mathbf{F}$

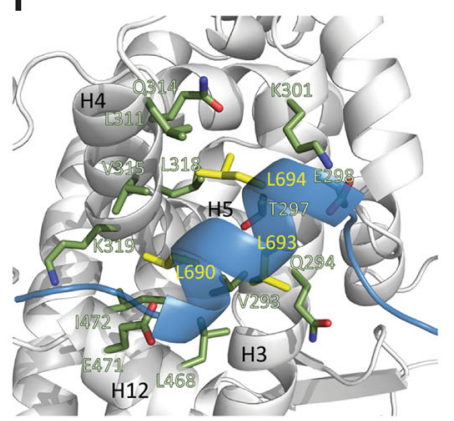

Fig. 6 Prediction of peptide/PPAR $y$ structures in silico. a Cartoon representation of PPARy with numbered helices. PDB code: $1 \mathrm{fm} 9^{45}$. b, c Surface representation of PPARY. Possible interaction sites coloured in red. The agonist-binding site, formed by residues of helices 3 and $2^{\prime}$, was predicted as a possible interaction site by the web server meta-PPISP (http://pipe.scs.fsu.edu/meta-ppisp.html, ref. ${ }^{37}$ ). The coactivator interaction site, including residues from helices 3, 4, 5 and 12, was predicted by the web server PINTS (http://www.russelllab.org/cgi-bin/tools/pints.pl, ref. ${ }^{42}$ ). Helix 10/11, the heterodimerization site, was predicted by coiled-coil predicting web servers DeepCoil (https://toolkit.tuebingen.mpg.de/\#/tools/deepcoil, ref. ${ }^{41}$ ) and Waggawagga (https://waggawagga.motorprotein.de, ref. ${ }^{40}$ ). The predicted interaction sites corresponded well with crystal structures of PPARY in complex with agonists, coactivator peptides and its coiled-coil interaction partner RXRa. $\mathbf{d}-\mathbf{f}$ Comparison of the modelled interactions of PEP (d), MP (e) and a SRC1-peptide (f, PDB code: 1fm9) with the coactivator interaction site. Cartoon representation of PPARy is coloured in white, helices are numbered in black and the residues interacting with the peptides are shown as sticks in green and labelled in white with green contour. Leucines of the LXXXL motif of PEP and of the LXXLL motif of SRC1 and MP are shown as yellow sticks and labelled in yellow, SRC1 and the peptides are represented as blue cartoon. For the PEP and MP peptides, interactions were calculated with AMBER1 $8^{67}$ from a $120 \mathrm{~ns}$ MD simulation. For SRC1, interactions were calculated from the $1 \mathrm{fm} 9$ structure. In both cases, the maximal interatomic contact distance was set to $5 \AA$, excluding hydrogens, and interactions were required to be present in more than $80 \%$ of the 50,000 frames analysed. The structure in the last frame of each simulation is shown.

with concentrations of rosi higher than its $\mathrm{IC}_{50}(>1 \mu \mathrm{M})$ reduced PPARY activity, presumably due to a "nongenomic" activation of the MEK1/2-ERK1/2-pathway and subsequent phosphorylation and inactivation of PPAR $\gamma^{16}$. Of note, exposure of cells to MP and PEP reversed this effect: In the presence of both peptides, increased PPAR $\gamma$ activity was detectable even after treatment with $10 \mu \mathrm{M}$ rosi (Fig. $5 \mathrm{~b}, " p<0.05$ vs. VC, two-way ANOVA, Holm-Sidak's multiple comparisons test, replicates: HEK293T: $n=7$, HCT116: $n=3$, SW480: $n=5$ ). To address the question whether this in vitro efficacy could be translated into the in vivo situation, C57BL6/J mice (pCEA-SV40-Tag) $^{34}$ were treated for 14 days with MP, PEP (both at $30 \mathrm{mg} / \mathrm{kg}^{*} \mathrm{~d}$ ) or vehicle control (VC) by intraperitoneal injection (four times per week), respectively. RT-qPCRs (Tab. S2) detecting exemplary PPAR $\gamma$ target genes were performed on total RNA extracted from snap-frozen whole tissue samples derived from the distal colon (Fig. 5c). P21 $1^{\text {(Cip1/Waf1) }}$ and Cd36 mRNAs were increased in animals treated with either of the peptides (VC: $n=4$ mice, MP: $n=8$ mice, PEP: $n=8$ mice, ${ }^{*} p<$ 0.05 vs. VC, Mann-Whitney test). This effect was accompanied by an increase in body weight (VC vs. PEP: $p=0.0112$; VC vs. MP: $p=0.1$, Mann-Whitney test) and spleen weight (VC vs. PEP: $p=0.0182$; VC vs. MP: $p=$ 0.0639, Mann-Whitney test) (Fig. 5d). Taken together, the MTMR7-CC mimicry peptides activated PPAR $\gamma$ in vitro and in vivo.

\section{Prediction of peptide/PPAR $y$ structures in silico}

To gain a better understanding of the potential molecular mechanism of the mimicry peptide mediated activation of PPAR $\gamma$, a computational approach was employed to generate plausible models of the peptide/ PPAR complexes. Both peptides (PEP and MP) were predicted to form $\alpha$-helical secondary structures by the web servers PredictProtein ${ }^{35}$ and Agadir $^{36}$ (Supplementary Figs. 2, 3). Therefore, they were both modelled as 
$\alpha$-helices, but the terminal myristoylation and the amide caps were omitted.

We then used three different web servers to identify putative peptide binding sites on the 12-helices bundle of the ligand-binding domain (LBD) of PPAR (Fig. 6a). This analysis yielded three candidate regions for interaction (Fig. 6b). The web server meta-PPISP ${ }^{37}$ predicted the residues surrounding the agonist-binding pocket of PPAR $\gamma$ to be the most likely interaction site on the protein ${ }^{38}$ (Fig. 6b), mainly formed by residues of helices $2^{\prime}$ and 3 and the loop between them ${ }^{39}$. The coiled-coil $(\mathrm{CC})$ predictors, Waggawagga $^{40}$ and DeepCoil ${ }^{41}$, predicted only helix $10 / 11$ as a possible site for coiled-coil interaction on PPAR $\gamma$. Helix 10 mediates heterodimerization between PPAR $\gamma$ and RXR $\alpha$ and forms a coiled-coil with helix 10 of RXR $\alpha$ (Fig. 6c). The PINTS web server ${ }^{42}$ yielded several crystal structures of PPAR $\gamma$ and related proteins with LXXLL motif-harbouring coactivators or corepressors ${ }^{43-46}$. The latter had been cocrystallized in an $\alpha$-helical conformation at the coactivator interaction site ${ }^{39}$ which is formed by helices $3,4,5$ and 12 of PPARY (Fig. 6b).

As the heterodimerization and the coactivator-binding sites were already experimentally shown to be binding sites for $\alpha$-helical peptides and protein regions, they were thought to be more likely interaction sites than the agonist-binding site. Docking was performed using two different web servers that employ different approaches: The web server GalaxyPepDock ${ }^{47}$ docked the MP exclusively with the canonical LXXLL motif at the coactivator binding site. The PEP was also docked exclusively to this site either with its LXXXL or LXXL motifs. The second tool used was the web server ClusPro ${ }^{48}$. The ClusPro docking resulted in complexes with the peptides docked to varying sites on PPAR $\gamma$, including helix 10/11 and the proximity of the coactivator interaction site.

To assess which of these complexes might be the most favourable ones, all-atom molecular dynamics (MD) simulations followed by calculations of binding free energies were performed on 23 complexes from the two web servers with MP and PEP bound to different sites on the nuclear receptor. Peptides were bound either to the heterodimerization site or the coactivator interaction site. The results of the free energy calculations indicated that the coactivator interaction site is a more favourable binding site than the heterodimerization site (Tab. S3). Interactions were calculated for the most energetically favourable complex between each of the peptides and PPAR $\gamma$, as well as for PPAR $\gamma$ with the classical coactivator peptide from SRC1. As seen in the last frame of the MD simulation, PEP bound to the coactivator interaction site with its N-terminal LXXXL motif (Fig. 6d), while MP and SRC1 bound to the interaction site with their canonical LXXLL motifs, respectively (Fig. 6e, f). Comparing the calculated interactions to those described by Nolte et al. ${ }^{39}$, the binding mode of MP seemed to be similar to SRC1, involving residues on helices 3,4 and 12 (Tab. S4), including K301 on helix 3 and E471 on helix 12 (Fig. 6e). These two residues have been described to form a charged clamp that is thought to position the coactivator motif on SRC1 in the correct orientation at the coactivator interaction site ${ }^{39}$.

PEP was not found to interact with residues on helix 12 in more than $80 \%$ of the $120 \mathrm{~ns}$ MD simulation and, therefore, is unlikely to make use of the charged clamp for positioning (Fig. 6d). The leucine at position five in the LXXLL/LXXXL motifs engaged in the majority of the interactions by inserting into a hydrophobic cleft between helices 3,4 and 5, as described for $\mathrm{SRC}^{39}$. For both peptides, this leucine was calculated to interact with F306 inside this cleft, an interaction residue not shown for SRC1.

The structures of the complete complexes of PPAR $\gamma$ with PEP or MP at the last frame of the MD simulations are shown in Supplementary Fig. 4. Outside the coactivator interaction site, their conformations differ. PEP was found to drape its $\mathrm{C}$-terminal end in a stable helix over the entrance of the agonist-binding pocket in proximity of helices $2^{\prime}$ and 3 , while its $\mathrm{N}$-terminus remained unstructured and did not form stable interactions. The $\mathrm{N}$ terminus of MP stayed close to helices 7 and 11, while its C-terminus was close to helix 1.

In conclusion, the in silico prediction of the structures of peptide/PPAR $\gamma$ complexes indicates a coactivatorlike binding mode that may explain the capacity of the peptides to stimulate the transcriptional activity of PPAR $\gamma$.

\section{Discussion}

In the present study, we describe a novel role for MTMR7 in human CRC, identifying it as a binding partner and positive regulator of PPAR $\gamma$. The transcriptional activity of PPARY is regulated by ligand binding, post-translational modifications and subcellular localisation ${ }^{49}$. The here described MTMR7-PPARY interaction complex offers a novel mode of PPAR $\gamma$ regulation by compartmentalization and a possible explanation for the pro- vs. anti-tumour effects of PPAR $\gamma$-agonists ${ }^{50-52}$. Previously, we characterized MTMR7 as an inhibitor of ERK1/2 and AKT/mTOR signalling ${ }^{25}$, thus acting as a dual blockage of two interconnected and compensatory oncogenic pathways downstream of receptor tyrosine kinases (e.g. the EGF receptor) and $\mathrm{RAS}^{53,54}$. MTMR7 may thus enforce the classical nuclear function of PPAR $\gamma$ by inhibiting the RAS-ERK $1 / 2$ cascade, which otherwise evokes inactivation of PPAR $\gamma$ by ERK1/2-mediated phosphorylation and MEK1/2-dependent sequestration of PPAR $\gamma$ in the cytosol ${ }^{16}$, where it interacts with molecules that restrain PPARY activity including caveolin- $1^{26}$ and heat shock proteins ${ }^{55}$. 

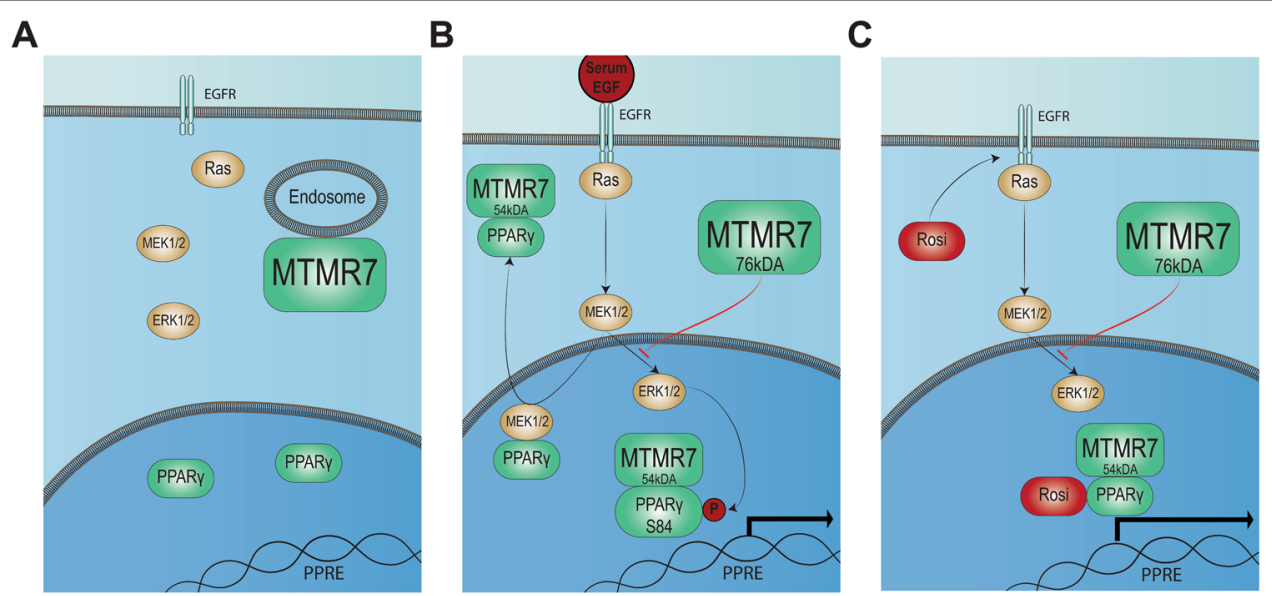

Fig. 7 MTMR7-PPARy signalling model. MTMR7 was identified as a novel PPARY interactor, which increased PPARy activity in vivo and in vitro. We propose a full-length $(\mathrm{FL})$ isoform $(76 \mathrm{kDa}$ ) in the cytosol, harbouring an N-terminal $\mathrm{PH}$ domain for binding to plasma and/or intracellular membranes (e.g. endosomes), and a mobile truncated isoform $(54 \mathrm{kDa})$, presumably consisting of the C-terminal part of MTMR7 harbouring the coiled-coil (CC) domain, which was also found in the nucleus. a In resting cells, MTMR7 is bound to endosomes (or other vesicular structures), and ERK1/2 and MEK1/ 2 reside in the cytosol, whereas PPARY is localized in the nucleus. $\mathbf{b}$ Upon stimulation with EGF (or serum), the kinases translocate into the nucleus where ERK1/2 phosphorylate PPARY on Ser84, while MEK1/2 export PPARY to the cytosol, two events resulting in inactivation of PPARY. We suggest that MTMR7 counteracts the inhibitory effects of the two kinases: (1) MTMR7 FL stays in the cytosol and/or attached to membranes via its PH domain and inhibits MEK1/2-dependent ERK1/2 activation by an yet unknown mechanism, presumably involving altered PIP metabolism and EGFR traffic as shown for other MTMs. This event abrogates post-translational inactivation of PPARy by the above mentioned kinases (in $\mathbf{b}$ ), resulting in increased transcriptional activity of PPARY. (2) Truncated MTMR7 translocates to the nucleus, directly binds to PPARy via its coiled-coil (CC) domain and acts as a nuclear receptor coactivator harbouring LXXLL-like motifs similar to SRC1. Thereby, the transcriptional activity of PPARY on target gene promoters is

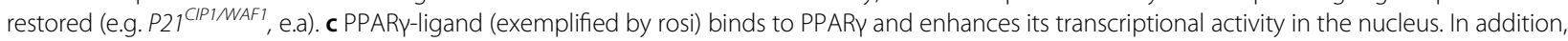
rosi triggers "non-genomic" trans-activation of plasma membrane receptors (e.g. EGFR, GPR40) and downstream kinases (in b). As for EGF/serum (in b), MTMR7 counteracts rosi-dependent "non-genomic" activation of MEKs/ERKs and promotes transcriptional activity of PPARY in the nucleus together with its mode of action as a SRC1-like coactivator.

In the current study, we provide evidence that MTMR7 also reduces the rapid "non-genomic activation" of ERK1/ 2 by the PPARy-agonist rosi ${ }^{56,57}$. Through this mechanism, which has been shown to be mediated by EGF- or Gprotein-coupled receptors (e.g. GPR40) in the plasma membrane, MTMR7 may prevent the adverse side effect of this class of PPAR $\gamma$-ligands on cell proliferation and organ damage ${ }^{49-52}$. This finding is of particular significance in light of the plethora of negative clinical trials using PPAR $\gamma$ agonists in the therapy of gastrointestinal malignancies ${ }^{14,58}$ : since the loss of MTMR7 is a common event in CRC, induced by stimuli including insulin or insulin-like growth factors ${ }^{25}$, the MTMR7 expression status might be a predictive parameter when targeting PPAR $\gamma$ in the setting of anti-proliferative therapies.

Moreover, we offer new insights into the subcellular distribution of MTMR7. While myotubularins in general are regarded to be cytosolic proteins ${ }^{21,22}$, we collected data suggesting that MTMR7 shuttles between the cytosol and the nucleus in response to stimuli of the RAS signalling cascade or to PPAR $\gamma$ agonist (here exemplary for rosi). In mice ${ }^{24}$, full-length (FL) $76 \mathrm{kDa}$ and truncated $54 \mathrm{kDa}$ isoforms of MTMR7 are expressed. The latter lacks the C-terminal coiled-coil (CC) domain and may thus be incapable of oligomerization with other
$\mathrm{MTMs}^{24}$. The peptides identified in SW480 cells by MALDI-MS also suggest the existence of a $54 \mathrm{kDa}$ isoform of MTMR7 in human CRC cells, forming the complex with PPAR $\gamma$. Western blot analyses using four different Abs against the C-terminal domain of MTMR7, as well as previously published exon-selective RT-PCRs from several human CRC cell lines ${ }^{25}$ confirmed the presence of truncated $<54 \mathrm{kDa}$ MTMR7 (Supplementary Fig. 5). We may hence conclude that the identified isoform is truncated at the $\mathrm{N}$-terminus, containing the $\mathrm{PH}$ domain, responsible for membrane association. This truncation might therefore enable MTMR7 to shuttle into the nucleus, together with its binding partner PPARY (see model in Fig. 7), to enforce the transcriptional activity of the nuclear receptor.

This mode of action is further supported by the observed efficacy of the MTMR7-CC mimicry peptide, resembling the coiled-coil (CC) domain of the phosphatase, which was able to activate PPAR $\gamma$ in vitro and in vivo: Notably, it induced transcription of PPAR $\gamma$ target genes as well as body weight gain, a well-known adverse effect attributed to PPAR $\gamma$ agonists of the thiazolidinedione class (e.g. rosi) ${ }^{49,59,60}$.

Exploitation of different web servers for binding site prediction and docking, in combination with MD 
simulations, pointed at the coactivator interaction site of PPARy being the most likely binding site for the peptides, similar to those of the bona fide coactivator SRC1, inserting the leucine at position 5 of its LXXLL motif into a hydrophobic cleft between helices $3,4,5$ and $12^{38}$. Complexes covering this interaction site were more stable and energetically favourable compared with any other candidate sites like the heterodimerization interface or the agonist-binding pocket. MP and PEP interacted with residues that were also reported for SRC1 by Nolte et al. $^{39}$. As such, MP made use of the charged clamp between E471 and K301 and interacted with several residues on helix 12 . Both peptides were also found to interact with F306, implicating that the LXXLL/ XXXL motifs might reach further into the cleft and contribute to the stable binding of the peptides to the coactivator interaction site. Notably, stretches of the peptides unfolded from their initial helical conformation assigned, whereas other parts, and especially the ones bound to the coactivator-binding site, stayed helical throughout the MD simulations. The stable helix formation of this peptide region agrees with reports on the behaviour of the LXXLL motif in SRC1 that is thought to be unstructured in its apo-form but to form a short amphiphatic $\alpha$-helix upon binding to the coactivatorbinding site ${ }^{61,62}$.

While the computational methods favour the coactivator interaction site and resulted in a seemingly plausible interaction prediction, one important short-coming of this model for the PPARY/PEP complex is that PEP does not contain the canonical LXXLL motif that was deemed to be necessary for this kind of coactivator-like interaction in the past ${ }^{62}$. The fact that MP gave more favourable binding free energies than PEP supports the reported importance of all three leucines. Cell-free competition assays with both peptides and SRC1 on recombinant PPAR $\gamma$-LBD protein would be necessary to experimentally confirm our in silico predictions.

Preliminary findings revealed that the peptides were unable to displace rosi from the PPAR $\gamma$-LBD, thus unlikely to act as true agonists; instead, rosi increased MTMR7/PPAR $\gamma$ complex formation and MTMR7 protein expression in CRC cells (not shown). These observations may indicate that, alike MTMR9, ligand-activated PPAR $\gamma$ could stabilize the MTMR7 protein, and, vice versa, MTMR7 may lead to a quasi-allosteric activation of PPARy via the SRC1 coactivator site. However, future in depth nuclear magnetic resonance (NMR) or cocrystallization studies will be required to experimentally prove the predicted in silico models.

Taken together, our study identified a novel positive regulator of PPAR $\gamma$ based on (i) inhibition of ERK1/ 2 signalling and (ii) direct interaction with the CC domain of MTMR7. Additional studies will be necessary to characterize the pharmacological properties of the peptides to allow further development of this novel PPARY activator for future clinical applications.

\section{Materials and methods \\ Reagents and plasmids}

Chemicals were from Merck/Sigma (Darmstadt, Germany). Antibodies (Abs) used are listed in Table. S2. Untagged and GFP-tagged PPAR $\gamma 1$ and PPRE-luc plasmids were mentioned previously ${ }^{15}$. Human full-length (FL) MTMR7 cDNA (start codon MEHIRT, aa 1-660, $76 \mathrm{kDa}$, NM_004686.4) was in pTarget (pT) vector (Promega GmbH, Mannheim, Germany) with or without GFP- $\operatorname{tag}^{25}$. Transient transfection and luciferase assays were performed as described ${ }^{63}$.

\section{Peptides}

PEP corresponded to the original leucine-rich aa sequence of MTMR7 (aa 521-550; SwissProt ID: Q9Y216.3: LMAVKEETQQLEEELEALEERLEKIQKVQL) (Tab. S1). MP was designed based on the PEP sequence using a random scrambling web tool (https://web.expasy. org/randseq/: VLQEEILEMTEEKALLQALLKKEQERVQEE) generating a canonical $L X X L L$ nuclear receptor coactivator motif. Both peptides were synthesized by automated, solid phase peptide synthesis on Rink amide resin and coupled at the $\mathrm{N}$-terminus with myristidic acid (ETH Zürich, Switzerland). The resulting peptides were characterised by HPLC and MALDI-MS for purity and sequence confirmation and provided as lyophilized powders $^{64}$.

\section{Cell culture}

Human embryonic kidney (HEK293T) and colon adenocarcinoma cell lines (all from the American Type Culture Collection, Rockville, MD) were maintained as before $^{26}$. PDOs from CRC tissues were cultivated as published in refs. ${ }^{65,66}$. All cultures were routinely tested for contamination with Mycoplasma (InVivogen, Toulouse, France).

\footnotetext{
Acknowledgements

We want to thank Frank Herweck, Olga Skabkina and Kauthar Srour for excellent technical assistance. This study was supported by grants to EB from the Deutsches Krebsforschungszentrum (DKFZ, German Cancer Research Center) and the Israel Ministry of Science and Technology (MOST) (Ca158) and from the Deutsche Krebshilfe (German Cancer Aid, \#108287; \#111086). J.B., P.W. and T.G. received support from the "Translational Physician Scientist" (TraPS) programme; L.H., T.S. and V.H. were awarded MD fellowships (all from the Medical Faculty Mannheim). A.N.A. was supported by a Capes-Humboldt fellowship from the Alexander von Humboldt Foundation and from the Coordenaçao de Aperfeiçoamento de Pessoal de Nivel Superior (Capes, process number $88881.162167 / 2017-01)$. D.S., A.N.A. and R.W. thank the Klaus Tschira Foundation for financial support. M.E. was funded by the State of Baden-Württemberg for the "Center of Geriatric Biology and Oncology (ZOBEL) Perspektivförderung" and "Biology of Frailty - Sonderlinie Medizin".
} 


\section{Author details}

'Department of Medicine II, Medical Faculty Mannheim, Heidelberg University, Mannheim, Germany. ²Department of Pathology, Christian-Albrechts University, Kiel, Germany. ${ }^{3}$ Department of Organic Chemistry, ETH Zürich, Zürich, Switzerland. ${ }^{4}$ Department of Biological Regulation, Weizmann Institute of Science, Rehovot, Israel. ${ }^{5}$ Heidelberg Institute for Theoretical Studies (HITS), Heidelberg, Germany. ${ }^{6}$ Zentrum für Molekulare Biologie der Universität Heidelberg (ZMBH), Heidelberg, Germany. ${ }^{7}$ Interdisciplinary Center for Scientific Computing (IWR), Heidelberg University, Heidelberg, Germany

\section{Author contributions}

All authors cooperated and contributed to, critically reviewed and approved the paper. E.B., M.E. and R.S. defined the research theme. E.B., L.H., M.S., P.W., T.G., T.S. and V.H. designed methods, carried out the experiments, analysed the data and interpreted the results. J.B. conducted sequencing and bioinformatics analyses. F.R., J.W.B. and V.P. synthesized the peptide. D.S. modelled 3D structures, performed and analysed simulations with the help and supervision of A.N.A. and R.W. C.R. provided clinical samples, performed and analysed immunohistochemical staining. D.S., E.B. and P.W. wrote the paper

\section{Conflict of interest}

The authors declare that they have no conflict of interest. Parts of the cell line data have been deposited under https://doi.org/10.11588/heidok.00025716; https://doi.org/10.11588/heidok.00023843.

\section{Publisher's note}

Springer Nature remains neutral with regard to jurisdictional claims in published maps and institutional affiliations.

Supplementary Information accompanies this paper at (https://doi.org/ 10.1038/s41389-020-0238-8).

Received: 3 December 2019 Revised: 15 April 2020 Accepted: 21 April 2020 Published online: 10 June 2020

\section{References}

1. Peters, J. M., Shah, Y. M. \& Gonzalez, F. J. The role of peroxisome proliferatoractivated receptors in carcinogenesis and chemoprevention. Nat. Rev. Cancer 12, 181-195 (2012).

2. Michalik, L., Desvergne, B. \& Wahli, W. Peroxisome-proliferator-activated receptors and cancers: complex stories. Nat. Rev. Cancer 4, 61-70 (2004).

3. Glass, C. K. \& Saijo, K. Nuclear receptor transrepression pathways that regulate inflammation in macrophages and T cells. Nat. Rev. Immunol. 10, 365-376 (2010).

4. Ogino, S. et al. Colorectal cancer expression of peroxisome proliferatoractivated receptor gamma (PPARG, PPARgamma) is associated with good prognosis. Gastroenterology 136, 1242-1250 (2009).

5. Maniati, E. et al. Crosstalk between the canonical NF-kappaB and Notch signaling pathways inhibits Ppargamma expression and promotes pancreatic cancer progression in mice. J. Clin. Invest. 121, 4685-4699 (2011).

6. Komatsu, Y. et al. Phase 1 study of efatutazone, a novel oral peroxisome proliferator-activated receptor gamma agonist, in combination with FOLFIRI as second-line therapy in patients with metastatic colorectal cancer. Invest. N. Drugs 32, 473-480 (2014).

7. Tebbutt, N., Pedersen, M. W. \& Johns, T. G. Targeting the ERBB family in cancer: couples therapy. Nat. Rev. Cancer 13, 663-673 (2013).

8. Karapetis, C. S. et al. K-ras mutations and benefit from cetuximab in advanced colorectal cancer. N. Engl. J. Med. 359, 1757-1765 (2008).

9. Cox, A. D., Fesik, S. W., Kimmelman, A. C., Luo, J. \& Der, C. J. Drugging the undruggable RAS: Mission possible? Nat. Rev. Drug Disco. 13, 828-851 (2014).

10. Cariou, B., Charbonnel, B. \& Staels, B. Thiazolidinediones and PPARgamma agonists: time for a reassessment. Trends Endocrinol. Metab. 23, 205-215 (2012).

11. Choi, W. et al. Identification of distinct basal and luminal subtypes of muscleinvasive bladder cancer with different sensitivities to frontline chemotherapy. Cancer Cell 25, 152-165 (2014).
12. Girnun, G. D. et al. Regression of drug-resistant lung cancer by the combination of rosiglitazone and carboplatin. Clin. Cancer Res. 14, 6478-6486 (2008).

13. Pishvaian, M. J. et al. A phase 1 study of efatutazone, an oral peroxisome proliferator-activated receptor gamma agonist, administered to patients with advanced malignancies. Cancer 118, 5403-5413 (2012).

14. Kulke, M. H. et al. A phase II study of troglitazone, an activator of the PPARgamma receptor, in patients with chemotherapy-resistant metastatic colorectal cancer. Cancer J. 8, 395-399 (2002).

15. Burgermeister, E. et al. Interaction with MEK causes nuclear export and downregulation of peroxisome proliferator-activated receptor gamma. Mol. Cell Biol. 27, 803-817 (2007).

16. Burgermeister, E. \& Seger, R. PPARgamma and MEK interactions in cancer. PPAR Res 2008, 309469-309484 (2008).

17. Banks, A. S. et al. An ERK/Cdk5 axis controls the diabetogenic actions of PPARgamma. Nature 517, 391-395 (2015).

18. von Knethen, A., Tzieply, N., Jennewein, C. \& Brune, B. Casein-kinase-Ildependent phosphorylation of PPARgamma provokes CRM1-mediated shuttling of PPARgamma from the nucleus to the cytosol. J. Cell Sci. 123, 192-201 (2010).

19. Burgermeister, E. \& Seger, R. MAPK kinases as nucleo-cytoplasmic shuttles for PPARgamma. Cell Cycle 6, 1539-1548 (2007).

20. $\mathrm{Ku}, \mathrm{Y} . \mathrm{H}$. et al. Rosiglitazone increases endothelial cell migration and vascular permeability through Akt phosphorylation. BMC Pharm. Toxicol. 18, 62-73 (2017).

21. Nicot, A. S. \& Laporte, J. Endosomal phosphoinositides and human diseases. Traffic 9, 1240-1249 (2008).

22. Hnia, K., Vaccari, I., Bolino, A. \& Laporte, J. Myotubularin phosphoinositide phosphatases: cellular functions and disease pathophysiology. Trends Mol. Med. 18, 317-327 (2012).

23. Zou, J. et al. Myotubularin-related protein (MTMR) 9 determines the enzymatic activity, substrate specificity, and role in autophagy of MTMR8. Proc. Natl Acad. Sci. USA 109, 9539-9544 (2012).

24. Mochizuki, Y. \& Majerus, P. W. Characterization of myotubularin-related protein 7 and its binding partner, myotubularin-related protein 9. Proc. Natl Acad. Sci. USA 100, 9768-9773 (2003).

25. Weidner, P. et al. Myotubularin-related protein 7 inhibits insulin signaling in colorectal cancer. Oncotarget 7, 50490-50506 (2016).

26. Burgermeister, E. et al. The Ras inhibitors caveolin-1 and docking protein 1 activate peroxisome proliferator-activated receptor gamma through spatial relocalization at helix 7 of its ligand-binding domain. Mol. Cell Biol. 31, 3497-3510 (2011)

27. Barretina, J. et al. The Cancer Cell Line Encyclopedia enables predictive modelling of anticancer drug sensitivity. Nature 483, 603-607 (2012).

28. Cerami, E. et al. The cBio cancer genomics portal: an open platform for exploring multidimensional cancer genomics data. Cancer Disco. 2, 401-404 (2012).

29. He, H. et al. Rosiglitazone causes cardiotoxicity via peroxisome proliferatoractivated receptor gamma-independent mitochondrial oxidative stress in mouse hearts. Toxicol. Sci. 138, 468-481 (2014).

30. Gardner, O. S., Dewar, B. J. \& Graves, L. M. Activation of mitogen-activated protein kinases by peroxisome proliferator-activated receptor ligands: an example of nongenomic signaling. Mol. Pharm. 68, 933-941 (2005).

31. Balla, T., Wymann, M. \& York, J. D. Phosphoinositides / Enzymes of Synthesis and Degradation. (Subcellular Biochemistry). (Springer Press, The Netherlands, 2012).

32. Kim, S. A., Vacratsis, P. O., Firestein, R., Cleary, M. L. \& Dixon, J. E. Regulation of myotubularin-related (MTMR)2 phosphatidylinositol phosphatase by MTMR5, a catalytically inactive phosphatase. Proc. Natl Acad. Sci. USA 100, 4492-4497 (2003).

33. Savkur, R. S. \& Burris, T. P. The coactivator $L X X L L$ nuclear receptor recognition motif. J. Pept. Res. 63, 207-212 (2004).

34. Thompson, J. et al. A transgenic mouse line that develops early-onset invasive gastric carcinoma provides a model for carcinoembryonic antigen-targeted tumor therapy. Int J. Cancer 86, 863-869 (2000).

35. Rost, B., Yachdav, G. \& Liu, J. The PredictProtein server. Nucleic Acids Res. 32 W321-W326 (2004)

36. Muñoz, V. \& Serrano, L. Development of the multiple sequence approximation within the AGADIR model of a-helix formation: comparison with Zimm-Bragg and Lifson-Roig formalisms. Biopolymers 41, 495-509 (1997).

37. Qin, S. \& Zhou, H. X. meta-PPISP: a meta web server for protein-protein interaction site prediction. Bioinformatics 23, 3386-3387 (2007). 
38. Chrisman, I. M. et al. Defining a conformational ensemble that directs activation of PPARgamma. Nat. Commun. 9, 1794-1809 (2018).

39. Nolte, R. T. et al. Ligand binding and co-activator assembly of the peroxisome proliferator-activated receptor-gamma. Nature 395, 137-143 (1998).

40. Simm, D., Hatje, K. \& Kollmar, M. Waggawagga: comparative visualization of coiled-coil predictions and detection of stable single alpha-helices (SAH domains). Bioinformatics 31, 767-769 (2015).

41. Ludwiczak, J., Winski, A., Szczepaniak, K., Alva, V. \& Dunin-Horkawicz, S. DeepCoil-a fast and accurate prediction of coiled-coil domains in protein sequences. Bioinformatics 35, 2790-2795 (2019).

42. Stark, A., Sunyaev, S., Russell, R. B. \& Russell, R. B. A model for statistical significance of local similarities in structure. J. Mol. Biol. 326, 1307-1316 (2003).

43. Sierra, M. L. et al. Substituted 2-[(4-aminomethyl)phenoxy]-2-methylpropionic acid PPARalpha agonists. 1. Discovery of a novel series of potent HDLc raising agents. J. Med Chem. 50, 685-695 (2007).

44. Chandra, V. et al. Structure of the intact PPAR-gamma-RXR- nuclear receptor complex on DNA. Nature 456, 350-356 (2008).

45. Gampe, R. T. Jr. et al. Asymmetry in the PPARgamma/RXRalpha crystal structure reveals the molecular basis of heterodimerization among nuclear receptors. Mol. Cell 5, 545-555 (2000).

46. $\mathrm{Xu}, \mathrm{H}$. E. et al. Structural basis for antagonist-mediated recruitment of nuclear co-repressors by PPARalpha. Nature 415, 813-817 (2002).

47. Lee, H., Heo, L., Lee, M. S. \& Seok, C. GalaxyPepDock: a protein-peptide docking tool based on interaction similarity and energy optimization. Nucleic Acids Res. 43. W431-435 (2015).

48. Kozakov, D. et al. The ClusPro web server for protein-protein docking. Nat Protoc. 12, 255-278 (2017).

49. Kawai, M. \& Rosen, C. J. PPARY: a circadian transcription factor in adipogenesis and osteogenesis. Nat. Rev. Endocrinol. 6, 629-636 (2010).

50. Lago, R. M., Singh, P. P. \& Nesto, R. W. Congestive heart failure and cardiovascular death in patients with prediabetes and type 2 diabetes given thiazolidinediones: a meta-analysis of randomised clinical trials. Lancet $\mathbf{3 7 0}$ 1129-1136 (2007)

51. Lefebvre, A. M. et al. Activation of the peroxisome proliferator-activated receptor gamma promotes the development of colon tumors in C57BL/6JAPCMin/+ mice. Nat. Med. 4, 1053-1057 (1998).

52. Lewis, J. D. et al. Risk of bladder cancer among diabetic patients treated with pioglitazone: interim report of a longitudinal cohort study. Diabetes Care 34, 916-922 (2011).
53. Shimobayashi, M. \& Hall, M. N. Making new contacts: the mTOR network in metabolism and signalling crosstalk. Nat. Rev. Mol. Cell Biol. 15, 155-162 (2015).

54. Hancock, J. F. Ras proteins: different signals from different locations. Nat. Rev. Mol. Cell Biol. 4, 373-384 (2003).

55. Wheeler, M. C. \& Gekakis, N. Hsp90 modulates PPARgamma activity in a mouse model of nonalcoholic fatty liver disease. J. Lipid Res. 55, 1702-1710 (2014).

56. Papageorgiou, E., Pitulis, N., Msaouel, P., Lembessis, P. \& Koutsilieris, M. The non-genomic crosstalk between PPAR-gamma ligands and ERK1/2 in cancer cell lines. Expert Opin. Ther. Targets 11, 1071-1085 (2007).

57. Luconi, M., Cantini, G. \& Serio, M. Peroxisome proliferator-activated receptor gamma (PPARgamma): Is the genomic activity the only answer? Steroids $\mathbf{7 5}$ 585-594 (2010).

58. Marshall, J. et al. A randomized, open-label phase II study of efatutazone in combination with FOLFIRI as second-line therapy for metastatic colorectal cancer (mCRC). J. Clin. Oncol. 32(Suppl 3), 535 (2014).

59. Ramakers, J. D. et al. The PPARgamma agonist rosiglitazone impairs colonic inflammation in mice with experimental colitis. J. Clin. Immunol. 27, 275-283 (2007).

60. Hiatt, W. R., Kaul, S. \& Smith, R. J. The cardiovascular safety of diabetes drugs-insights from the rosiglitazone experience. N. Engl. J. Med. 369 1285-1287 (2013).

61. Plevin, M. J., Mills, M. M. \& Ikura, M. The LxxLL motif: a multifunctiona binding sequence in transcriptional regulation. Trends Biochem Sci. $\mathbf{3 0}$ 66-69 (2005).

62. Heery, D. M., Kalkhoven, E., Hoare, S. \& Parker, M. G. A signature motif in transcriptional co-activators mediates binding to nuclear receptors. Nature 387, 733-736 (1997).

63. Burgermeister, E. et al. A novel partial agonist of peroxisome proliferatoractivated receptor-gamma (PPARgamma) recruits PPARgamma-coactivator1 alpha, prevents triglyceride accumulation, and potentiates insulin signaling in vitro. Mol. Endocrinol. 20, 809-830 (2006).

64. Zhang, Y. et al. Chemical synthesis of atomically tailored SUMO E2 conjugating enzymes for the formation of covalently linked SUMO-E2-E3 ligase ternary complexes. J. Am. Chem. Soc. 141, 14742-14751 (2019).

65. Zhan, T. et al. MEK inhibitors activate Wnt signalling and induce stem cell plasticity in colorectal cancer. Nat. Commun. 10, 2197-2213 (2019).

66. Betge, J. et al. Multiparametric phenotyping of compound effects on patient derived organoids. bioRxiv https://doi.org/10.1101/660993 (2019).

67. Case, D. A. et al. AMBER 2018. (University of California, San Francisco, 2018). 